\title{
CRITICAL INCIDENTS IN PAEDIATRIC ANAESTHESIA: DO THEY EXIST IN SPAIN TOO?
}

\section{Romera A, Garrido A, Cabrerizo P, De Miguel A, López-Gil M, Bravo C}

Gregorio Marañón University Hospital, Madrid [Spain]

This study reports the Spanish experience in critical incidents observed in paediatric anesthesia from January 2009 to July 2015.

\section{MATERIAL AND METHODS}

- Since 2009, the Anaesthesia and Intensive Care National Incident Reporting System [SENSAR] has analysed more than 5.000 critical incidents regarding patient safety and suggested more than 9.900 corrective measures based on the data collected.

- More than 80 Spanish hospitals and more than 380 members are part of SENSAR nowadays.

- SENSAR collects data from a confidential, anonymous, voluntary and non-punitive online reporting system [ANESTIC].

- A Critical Incident is defined as any event that affected, or could have affected, the safety or the quality of care of the patient whilst under the care of a health system.

- Data is analysed by a local team of experts, following the Protocol for the Investigation and Analysis of Clinical Incidents, first published in 1999 by Vincent.

- The analysis is focused on the system (rather than the individual] to generate a set of improvement strategies.

- All incidents and measures are shared online, so the other hospitals enrolled can learn from them.

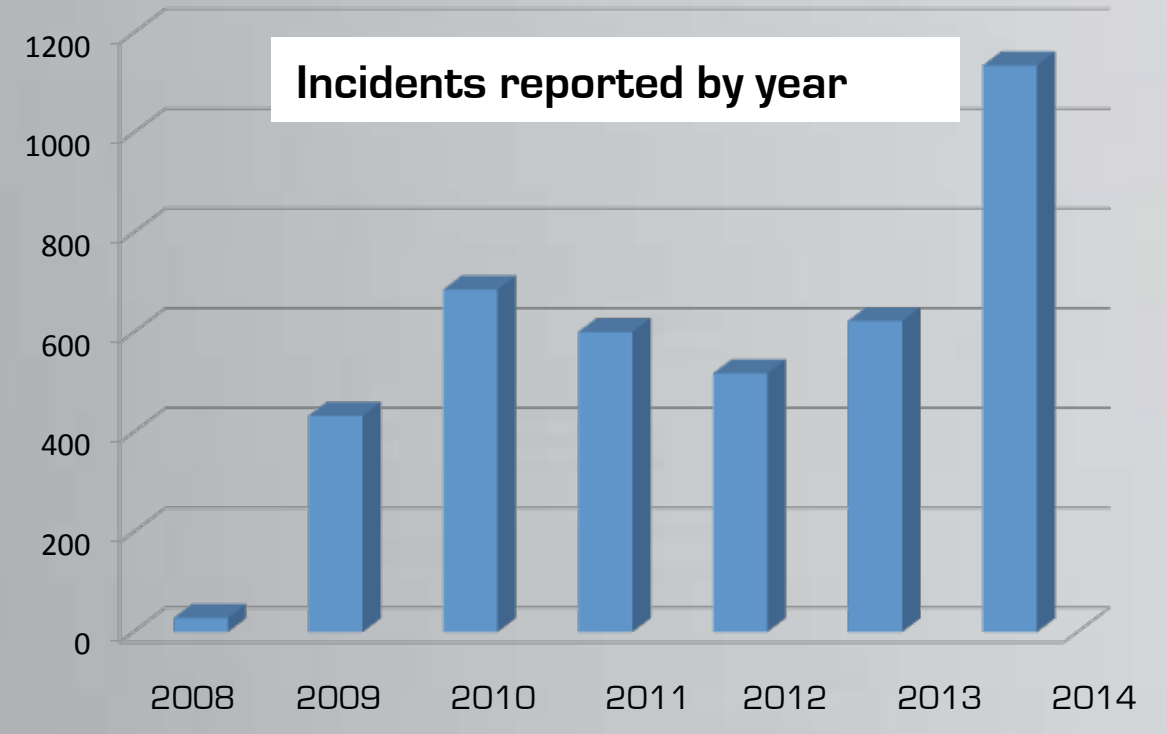

\section{RESULTS}

- 154 incidents in children up to 16 years old were reported.

- SENSAR's incidence of paediatric reporting is 2,8\%

- 122 were healthy ASA I-II.

- 51 of them took place in children under the age of 2 .

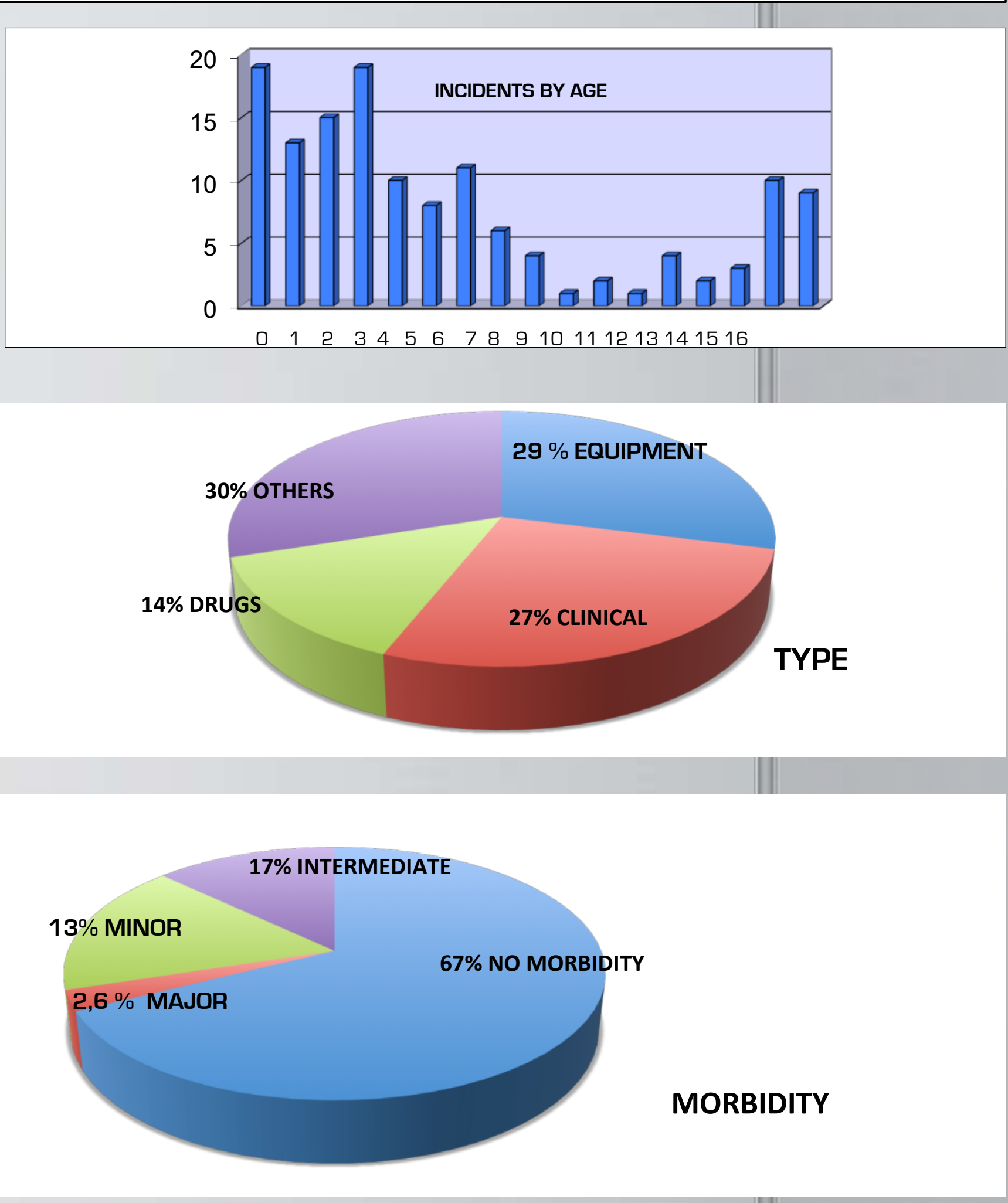

- No deaths were reported.

- Corrective measures were adopted in 50\% of the major morbidity cases as a result of the reported incidents.

\section{LIMITATIONS:}

- Only 25 hospitals [30\% of the total enrolled] reported paediatric incidents.

- The online database does not give any information regarding type of patients or type of care provided by each hospital.

\section{CONCLUSIONS}

- Children under one year of age have a higher risk of suffering a critical incident.

- Equipment and clinical [respiratory] incidents are the most common ones.

- Although it needs further improvement, the database is a useful tool that provides insight in the healthcare system and enhances patient safety.

- Paediatric anaesthetists should be encouraged to take ownership and report to national systems. 